\title{
Erratum to: A new hereditary colorectal cancer network in the Middle East and eastern Mediterranean countries to improve care for high-risk families
}

\author{
Zeinab Ghorbanoghli ${ }^{1}$ - Carol Jabari $^{2,19} \cdot$ Walid Sweidan $^{3} \cdot$ Wail Hammoudeh $^{4} \cdot$ George Cortas $^{5}$ - Ala I. Sharara ${ }^{6}$.

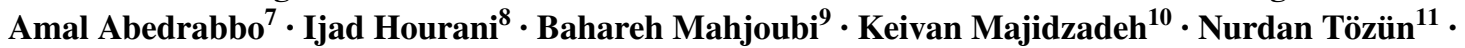 \\ Hadia Ziada-Bouchaar ${ }^{12}$ - Waseem Hamoudi ${ }^{13}$. Osama Diab ${ }^{14} \cdot$ Hamid Reza Khorram Khorshid $^{15}$. \\ Henry Lynch ${ }^{16} \cdot$ Hans Vasen ${ }^{17,18}$
}

Published online: 27 July 2017

(C) Springer Science+Business Media B.V. 2017

\section{Erratum to: Familial Cancer DOI 10.1007/s10689-017-0018-6}

Unfortunately, the 10th author name in the author group was published incorrectly in the original publication as "Keivan Maijdzadeh" the correct name is: Keivan Majidzadeh.

The online version of the original article can be found under doi:10.1007/s10689-017-0018-6.

\section{Hans Vasen}

hfavasen@gmail.com

1 Department of Gastroenterology and Hepatology, Leiden University Medical Centre \& Netherlands Foundation for the Detection of Hereditary Tumours, Leiden, The Netherlands

2 Patient's Friends Society, Jerusalem, Palestine

3 Department of Gastroenterology, Makased Islamic Charitable Hospital, Jerusalem, Palestine

4 Department of Internal Medicine, Arabcare Hospital, Ramallah, Palestine

5 Department of Gastroenterology, St. George Hospital Medical Center, University of Balamand Medical School, Beirut, Lebanon

6 Division of Gastroenterology, American University of Beirut Medical Center, Beirut, Lebanon

7 Department of Pediatrics, Makased Islamic Charitable Hospital, Jerusalem, Palestine

8 Department of Surgery, Agusta Victoria Hospital, Jerusalem, Palestine

9 Colorectal Research Center, Iran University of Medical Sciences, Tehran, Iran
Author name has also been corrected in the original publication.

10 Motamed Cancer Institute, ACECR, Tehran, Iran

11 Department of Internal Medicine and Gastroenterology, University of Acibadem, Acibadem Kozyatagi Hospital, Istanbul, Turkey

12 Laboratory of Biology and Molecular Genetics, Faculty of Medicine, University 3, Constantine, Algeria

13 Department of Gastroenterology, The Royal Hospital, Amman, Jordan

14 Department of Internal Medicine, Creighton University, Omaha, USA

15 Genetic Research Center, University of Social Welfare and Rehabilitation Sciences, Tehran, Iran

16 Creighton's Hereditary Cancer Center, Creighton University, Omaha, USA

17 Department of Gastroenterology \& Hepatology, Leiden University Medical Centre, P.O. Box 9600, 2300 RC Leiden, The Netherlands

18 Netherlands Foundation for the Detection of Hereditary Tumours, Leiden, The Netherlands

19 Hebron University, Hebron, Palestine 